ORIGINAL ARTICLE

\title{
Effect of Pulley Therapy on Genurecurvatum in Children with Unilateral Cerebral Palsy: Randomized Controlled Trial
}

\author{
MERHAN M. HAMOUDA ${ }^{1 *}$, NANEES E. MOHAMED ${ }^{2}$, WALAA A. ABD EL-NABIE ${ }^{2}$ \\ Faculty of Physical Therapy, Cairo University, Cairo, Egypt \\ ${ }^{1 *}$ Department of Physical Therapy, Cairo university, Toukh Health Management Restrict, Toukh, El Qalubia, Egypt. \\ ${ }^{2}$ Department of Physical Therapy for Pediatrics, Faculty of Physical Therapy, Cairo University, Giza, Egypt. \\ Corresponding author: Merhan M. Hamouda \\ Email : merhan.megahid1992@gmail.com,Tel : 01128271313 \\ Corresponding address: 7 Ahmed Elzayat st. Been Elsarayat, PO 12624, Dokki, Giza, Egypt.
}

\begin{abstract}
Objectives: Genu recurvatum commonly represents a more challenging problem that interferes with activities of daily living in children with cerebral palsy. This study aimed to investigate the effect of pulley therapy on genu recurvatum in children with unilateral cerebral palsy.

Methods: Forty children with unilateral cerebral palsy aged from 3 to 6 years were randomly allocated into two equal groups: control group and study group. Both groups received designed physical therapy program in addition, study group received pulley therapy program instead of using free weights in control group for strengthening hamstring and tibialis anterior muscles. The program's protocol applied for one hour, three times per week for three successive months for each group. Degree of genu recurvatum was assessed pre- and post-treatment by using digital goniometer while, muscle strength of hamstring and tibialis anterior was assessed by using Lafayette manual muscle tester device.

Results: Both groups showed significant improvement of all measured variables post treatment. The significant improvement after treatment in degree of genu recurvatum and muscle strength was obtained in favor to study group $(\mathrm{p}<0.05)$.

Conclusions: Pulley therapy may be used within the rehabilitation program of children with unilateral cerebral palsy to treat genu recurvatum.

Key words: Unilateral cerebral palsy, Hemiplegia, Genu recurvatum, Progressive resistance training, Pulley therapy.
\end{abstract}

\section{INTRODUCTION}

Cerebral palsy (CP) describes a group of neurological disorders that causes permanent damage of motor brain areas. It is due to lesion in immature brain during fetal or infancy resulting in sensory and motor deficits along with mental retardation, speech impairment and hearing problems [1]

Unilateral cerebral palsy (Hemiplegic CP) is a subtype of spastic CP in which upper and lower extremities on the same side of the body are affected. Muscle spasticity on the affected side limits the muscle and bone growth resulting in decreasing range of motion (ROM) [2].

About $20-30 \%$ of people with CP have unilateral cerebral palsy (UCP). For the hemiplegic child, spasticity and weakness are problems at the level of body function ${ }^{[3]}$. Children with UCP have different patterns of gait ${ }^{[4]}$. The pattern of the abnormal gait is affected by brain lesion primarily, secondary deformities, or compensatory mechanisms. One of these patterns is knee recurvatum ${ }^{[5]}$.

Genu recurvatum (GR) defined as a deformity of the tibio-fibular joint, in which the ROM exceeds zero degree of extension. $40-68 \%$ of ambulating hemiplegic children walk with genu recurvatum ${ }^{[6]}$. When left without treatment, it may cause laxity of knee posterior structures leading to early knee degeneration causing pain, deformities, instability and decreased independence in activities of daily living (ADL) ${ }^{[7]}$.

It is caused by limitation in ankle dorsal flexion due to increased tone and/or retraction of the posterior leg muscles and it is reported in children with UCP. As a result, during the stance phase the tibia is unable to move forward putting the knee in hyperextension position, as a result of ankle stiffness. Another cause of GR is weakness of knee flexors (hamstring muscles), during the stance phase hamstring muscles contraction is important for the knee flexion control-especially in the presence of knee extensor spasticity ${ }^{[8]}$.

Pulley therapy is a part of universal exercise unit which formed of spider cage and a system of pulley, straps and weights for resistance. It is a new method used for strengthening of weak muscles by isolating the target specific muscle for training ${ }^{[9]}$. There are several benefits of pulley therapy as: improving passive/active ROM, flexibility of muscle, enhancing strength and endurance of muscle without any associated movement and improving functional skills and dynamic movement ${ }^{[10]}$.Therefore, the aim of this study was to investigate the effect of pulley therapy on genu recurvatum in children with UCP. It was hypothesized that pulley therapy may improve hamstring and tibialis anterior muscles strength and decrease the degree of genu recurvatum.

\section{METHODOLOGY \\ Participants}

Forty UCP children from both sex accepted to participate in the current study, their ages were from three to six years. They were selected from Banha Educational Hospital and Al Safwa Outpatient Clinic for Pediatric Physical Therapy. Their degree of spasticity of lower limb muscles was 1 or $1+$ according to Modified Ashworth Scale [11]. They were at level I and II on Gross Motor Function Classification Scale (GMFCS) [12], had functional genu recurvatum and can 
follow orders and instructions of both evaluation and treatment procedures. Children were excluded if they exhibited visual or significant perceptual problems, severe limb deformities, surgical interference in lower limb, or botulinum toxin injection of the spastic lower limb's muscles during the last six months.

Approval of this study by the Faculty of Physical Therapy, Cairo University Ethical Committee (PT/REC/ 012/002127) and informed consent from all children' parents have been obtained before starting the study.

\section{Design and Randomization}

The current study was a randomized controlled trial which carried out from March 2020 to December 2020. Fortythree UCP children were assessed to take part in this study. Three children were excluded because they failed to fulfill the inclusion criteria. The remaining forty children were randomly assigned into two groups; control and study group. Both groups received designed physical therapy program, in addition, study group received pulley therapy program for strengthening hamstring and tibialis anterior muscles instead of using free weights in control group. Randomization was implemented by closed sealed envelopes, a paper card of either study or control group introduced in each envelop. This process occurred by an independent one who unaware by the study aim or protocol. Flow chart for the selection of participants represented according to Consolidated Standards of Reporting Trials (CONSORT) (Figure 1).

\section{Sample size}

Estimated sample size prior to the study using G*POWER statistical software (version 3.1.9.2; Franz Faul, Universitat Kiel, Germany) using $\alpha$ level $0.05, \beta=0.2$ and effect size $=0.91$. indicated that 20 subjects per group was required with ratio $\mathrm{N} 2 / \mathrm{N} 1=1$.

\section{PROCEDURES}

\section{A- Evaluative procedures}

In the current study degree of genu recurvatum and muscle strength of hamstring and tibialis anterior were assessed before and after treatment by using digital goniometer and Lafayette manual muscle tester respectively.

1. Evaluation of degree of genu recurvatum: Digital goniometer (model: 12-1027, White Plains, New York, USA) is a device that measures an angle and range of movement of a joint. It is affordable and easy to use for single or repeatable measurements. It was proved to be a highly reliable tool for measuring joints ROM in children with $\mathrm{CP}[13]$.

Each child lied in supine maintaining neutral hips and knees position. The examiner considered the femur lateral epicondyle as a fulcrum of goniometer while, the stationary and the movable arms of the goniometer were placed directed to greater trochanter in parallel to the femur's long axis and to lateral malleolus in parallel to the fibula's long axis respectively. Two adhesive straps were used to stabilize each arm of the device. Each child was asked to raise his foot upward while thigh was stabilized. Degree of motion was taken from the digital goniometer screen [14].

2. Evaluation of muscle strength: Lafayette manual muscle tester (MMT) (model: 01163, Parkway North, USA) is a new ergonomic design that has precision and accuracy and gives an objective, reliable and more accurate results.
It is hand held strength measurement system that designed for high inter and intra instrument reproducibility, automatic or manual storage of data and minimal measurement drift. Both peak force and time recorded so that muscle strength results were stable, and more accurate and reliable [15]. Test's procedures were demonstrated to each child. For both tests each child performed three corrected trails then the average was taken in analysis.

For hamstring muscle: Each child seated on a chair with hips and knees flexed $90^{\circ}$ and hands rested on thighs. The examiner stabilized trunk, pelvis and unexamined limb by straps to prevent any substitutions. The examined limb stabilized above knee by examiner's one hand and in the other hand Lafayette device placed. The examiner put the device on the posterior lower part of the examined leg and asked the child to move his/her lower leg backward against the device ${ }^{[16]}$.

For tibialis anterior muscle: Each child lied supine with feet relaxed on the plinth. Hips and knees were in extension and neutral rotation. The examiner stabilized the child by straps at pelvis and just above knees, then placed the device in her hand and put it on the dorsum aspect of examined foot over the metatarsal heads and asked the child to take his foot toward him against device ${ }^{[16]}$.

\section{B) Treatment procedures}

The treatment protocol for all participated children was carried out at Al Safwa Outpatient Clinic for Pediatric Physical Therapy.

For control group: Each child of control group received a designed physical therapy program, attending three sessions per week for three consecutive months. The duration of each session was one hour that was divided into half an hour for exercises to improve motor function, balance and gait and half an hour for strengthening of hamstring and tibialis anterior muscles by using free weights (adjustable weight cuffs attached by Velcro straps to the child lower leg). The program used a combination of open-and closed-chain exercises. Exercises for facilitating standing and balance as kneeling and half kneeling exercises, step standing, single limb support, from supine to stand, from prone to stand and standing on balance board, abdominal and core stability exercise on wedge, exercises for strengthening of hamstring and tibialis anterior muscles by using free weights and gait training including walking in stepper between parallel bars and ascending and descending stairs [17].

For study group: Each child in the study group received the same program as control group in addition to pulley therapy, attending three sessions per week for three consecutive months. The duration of the session was one hour that was divided into half an hour for the designed physical therapy program and half an hour for the pulley therapy. The pulley therapy is used to strength hamstring and tibialis anterior muscles.

The weight used in training is determined by using the one repetition maximum method (I-RM). The 1-RM was taken as the maximum resistance that could be lifted throughout the full ROM. To assess 1-RM, estimate the starting weight to be a little bit less than the child's maximum capacity for lifting. Then for exercises, weight increased gradually in each attempt till the child reached maximum lifting capacity. The added weight ranged from 1 
to $5 \mathrm{~kg}$ according to the evaluated muscle group. With periods of rest from 1 to 5 minutes allowed before attempting a lift at the next heavier weight ${ }^{[18]}$. Children performed a mild to moderate load (about $60 \% 1-\mathrm{RM}$ ) for 1 to 2 sets with $8-15$ repetitions ${ }^{[19]}$.

For hamstring muscle: each child lied prone on the table inside spider cage. The child stabilized by straps at pelvis and unaffected limb. The affected limb was stabilized at thigh level with lower leg free to move. A band around lower leg attached to upper part of the cage by a rope at foot side. At the end of the rope there was a weight to pull. Each child was asked to pull his leg toward him against weight doing one set of 10 repetitions at first up to 1-2 sets of $8-15$ repetitions.

For tibialis anterior: each child lied supine on the table inside spider cage. The child stabilized by straps at pelvis and unaffected limb. The affected limb was stabilized by straps at thigh and lower leg with foot free to move. A band around foot attached to upper part of the cage by a rope at foot side. At the end of the rope there was a weight to pull. Each child was asked to pull his foot toward him against weight doing one set of 10 repetitions up to $1-2$ sets of $8-15$ repetitions.

Statistical methods: Descriptive statistics and unpaired ttest were used to compare between groups means and standard deviations of subject's characteristics. MannWhitney test was conducted to compare GMFCS and Chisquared was carried out for comparison of sex and spasticity grades distribution between groups. Unpaired ttest was performed to compare the mean values of degree of genu recurvatum and tibialis anterior and hamstring muscles strength between the control and study groups. For comparison between pre and post treatment in each group Paired t-test was used. For all statistical tests the significance level was $p<0.05$. The analysis was done using the statistical package for social studies (SPSS) version 25 for windows (IBM SPSS, Chicago, IL, USA).

\section{RESULTS}

\section{Subject characteristics}

Table (1) showed the subject characteristics of the study and control groups. There was no significant difference in the mean age, weight, height, GMFCS, sex and spasticity grade distribution between groups $(p>0.05)$.

Effect of treatment on degree of genu recurvatum and tibialis anterior and hamstring muscles strength:

-Within group comparison: There was a significant decrease in the degree of genu recurvatum post treatment compared with that pre-treatment in the study and control groups $(p=0.001)$. The percent of decrease in the degree of genu recurvatum in the control group was $9.79 \%$ while that in the study group was $19.06 \%$. There was a significant increase in tibialis anterior and hamstring muscles strength post treatment compared with that pretreatment in both groups $(p=0.001)$. The percent of increase in tibialis anterior and hamstring muscles strength in the control group were 16.59 and $14.44 \%$ respectively while that in the study group were 32.47 and $26.32 \%$ for tibialis anterior and hamstring muscles strength respectively. (Table 2)

Between group comparison: There was no significant difference in degree of genu recurvatum and tibialis anterior and hamstring muscles strength between both groups pre-treatment $(p>0.05)$. Comparison between groups post treatment revealed a significant decrease in degree of genu recurvatum of the study group compared with that of the control group $(p<0.05)$. Also, there was a significant increase in tibialis anterior and hamstring muscles strength of the study group compared with that of the control group $(p<0.05)$. (Table 2$)$

\section{DISCUSSION}

Genu recurvatum may be caused by several factors as weakness of the muscles, impairment in muscle tone and joint proprioception deficits leading to poor knee joint control. During ambulation uncontrolled knee locking leads to recurrent micro trauma which results in instability and degenerative changes ${ }^{[20]}$. So, management of genu recurvatum should include correcting biomechanical faults and muscle imbalances.

The current study assessed the effect of pulley therapy on genu recurvatum in children with UCP. Our study set out to find whether strengthening of hamstring and tibialis anterior by pulley therapy would reduce the recurvatum of the knee. The protocols of treatment was completed adequately by both groups resulting in a significant within group improvement in the strength of hamstring and tibialis anterior muscles and decrease in the degree of knee recurvatum when comparing post treatment with pre-treatment. All measured outcomes showed significant improvement post treatment in the study group via achieving protocols of treatment more than control group.

Selection of age of participants in this study was from 3 to 6 years due to the ability of children at this age group to perform strengthening exercises which supported by Fry et al. ${ }^{[21]}$ who documented that children from 3-7 years of age are capable of performing strength and power tests and training.

The improvement of knee recurvatum of the study group using pulley therapy may be because of the impact of pulley therapy on hamstring and tibialis anterior strength through creating dorsiflexion on the foot which in turn create flexion on the knee hence reducing the degree of recurvatum during standing and this comes in agreement with Elnahas et al. ${ }^{[22]}$ who revealed that pulley therapy may be used in children with spastic CP to improve balance and lower limb muscles strength by improving neural function, muscle architecture and increasing cross sectional area of muscle, therefore improving standing ability and balance in children with spastic $\mathrm{CP}$.

Also, this comes parallel to the work of Mohamed et al. ${ }^{[23]}$ who reported that pulley therapy training may be a valuable additional tool for rehabilitation in children with spastic CP and it is a theoretically feasible method to improve muscle strength, gait and functional skills.

The improvement of the all measured outcomes of the control group suggested benefits from a well designed physical therapy program due to its effect on increasing muscle strength of hamstring and tibialis anterior which in turn led to decreasing genu recurvatum and this came with agreement of Morton et al. ${ }^{[24]}$ who revealed that physical therapy program including progressive resistance training 
led to increased strength of knee muscles which in turn result in improving gait and walking stability.

The significant decrease in degree of knee recurvatum post treatment in the study group showed that, pulley therapy may improve knee recurvatum. This came in agreement with study of Ahmed et al. ${ }^{[25]}$ on thirty hemiplegic CP revealed that adding loads around ankle joint during gait increase dorsiflexor strength and significantly increase knee flexion and improve gait function. Also, Shamay et al. [26] revealed that the rehabilitation program of the hemiplegic patient should include strengthening exercises to ankle dorsiflexors for better gait function. These support our result that strengthening ankle dorsiflexors by using pulley therapy can improve genu recurvatum.

The current study has some limitations such as it was done on one type of $\mathrm{CP}$ and their age was from three to six. The selected sample was restricted to level 1 or 2 GMFCS. So, further studies are needed to be done on other CP types, other types of neurological disabilities and other age group. Additionally, similar studies are needed to be done on other muscle groups as hip extensors and its effect on genu recurvatum in children with UCP.

\section{CONCLUSION}

The findings of this study were that significant intervention effects were found for children with UCP of two groups, and subjects of study group showed more favorable effects than subjects of control group in improving hamstring and tibialis anterior muscles strength and therefore decreasing degree of genu recurvatum.

Acknowledgments: Authors thank all children and their parents for participation in this study.

\section{REFERENCES}

1. Rosenbaum, P., Paneth, N., Leviton, A., Goldstein, M., Bax, M., Damiano, D, Dan B, Jacobsson, B. (2007). A report: the definition and classification of cerebral palsy April 2006. Developmental Medicine \& Child Neurology Supplement, 109: 8-14.

2. Yarnell, J. 2013. Epidemiology and disease Prevention: A Global Approach In: PRESS, O. U. (ed.) $2^{\text {nd }}$ ed. Oxford University Press: Oxford University.

3. Graham H.K., Rosenbaum P., Paneth N., et al. Cerebral palsy. Nat. Rev. Dis. Primers. 2016;2:15082. Doi:10.1038/nrdp.2015.82.

4. Senst, S. Unilateral spastic cerebral palsy (Hemiparesis). Der Orthopade 2014, 43, 649-655.

5. Agostini V, NascimbeniA, Gaffuri A and Knaflitz M. Multiple gait patterns within the same winter's class in children with hemiplegic cerebral palsy. Clin. Biomech.2015, 30, 908-914.

6. Sutherland DH and Davids JR. Common gait abnormalities of the knee in cerebral palsy. Clin Orthop Relat Res 1993:288:139-147.

7. Kerrigan DC, Deming LC and Holden MK. Knee recurvatum in gait: a study of associated knee biomechanics. Arch Phys Med Rehab 1996;77(7):645-50.

8. Vescguren O, Maltais DB, Gorter JW., Et al. Muscle strengthening in children and adolescent with spastic cerebral palsy: considerations for future resistance training protocols. Phys Ther. 2001; 91(7):1130-1139.

9. Liaqat $S$, Butt $M$ and Javaid HMW. Effects of universal exercise unit therapy on sitting balance in children with spastic and athetoid cerebral palsy Khyber Med Univ J 2016; 8(4): $177-180$

10. Afzal $F$, Asim H, Rasul A and Islam A. Effects of universal exercise unit combined with conventional combination therapy on gross motor and functional skills in spastic and athetoid cerebral palsy children. International Journal Medicine Appl. Health. 2015;3(1):28-34.

11. Alshryda S, Wright J. Development and reliability of a system to classify gross motor function in children with cerebral palsy. In: Classic Papers in Orthopaedics. Springer; 2014:575-577.

12. Damiano DL, Doddk J. and Taylor NF.: Should we be testing and training muscle strength in cerebral palsy? Dev. Med. Child Neurol.2002,44:68-72. 10.1017/S00126201001682.

13. Carey MA, Laird DE, Murray KA and Stevenson JR. Reliability, validity and clinical usability of a digital goniometer. National Library of Medicine 2010;36(1):55-66. Doi: 10.3233/WOR-2010-1007. PMID:20555176.

14. Hancock GE, Hepworth T, Wembridge K. Accuracy and reliability of knee goniometry methods. J Exp Orthop. 2018;5:46. doi:10.1186/s40634-.018-0161-5.

15. Mentiplay BF, Perraton L, Bower KJ and Adair B. Assessment of lower limb muscle strength and power using hand-held and fixed dynamometry 2015 Oct 28;10(10):e0140822.doi:10. 1371/journal .pone.0140822.

16. Bohannon RW. Test-retest reliability of hand-held dynamometry during a single session of strength assessment. Phys Ther. 1986;66(2):206-8.

17. Madbouly MM, Olama KA, Ayoub HS and Hachem RH. Knee cage versus long ankle foot orthosis for genurecurvatum in children with spastic diplegia 2018. 22-23 March. The $19^{\text {th }}$ International Scientific Conference Faculty of Physical Therapy; $1-8$.

18. Avery DF, Laurie AM and Wayne LW. Maximal strength testing in healthy children Journal of Strength and Conditioning Research. 2003; 17(1):162-166.

19. David G, Avery DF, Falk B and Klentrou P. Canadian Society for Exercise Physiology position paper: resistance training in children and adolescent. Physiol. Nutr. Metab. 2008; 33:547-561.

20. Sherief AA and Hamed SA. Electrical stimulation versus rebounding exercise on the degree of genu recurvatum in children with central hypotonia. Life Science Journal 2013;10(1):1724-1728.

21. Fry AC, Irwin CC, Nicoll JX and Ferebee DE. Muscular strength and power in 3-to-7-years-old children. Pediatr Exerc Sci 2015;27(3): 345-54.

22. El nahas AM, Olama KA, Rajab SH and Nour EI din SM. Effect of universal exercise unit on balance in children with spastic diplegia. International Journal of Multidisciplinary Research and Development. Volume6;Issue 1;January 2019;Page No.85-88

23. Mohamed AS, Kamal HM, Elsied AO and Abd El-Gafar EM. Universal Exercise Unit versus functional resisted training effect on muscle strength in spastic diaplegia Apr2020, 11(4): 1139-1144.

24. Morton JF, Brownlee M and Mcfadyen AK. The effects of progressive resistance training for children with cerebral palsy. Clinical Rehabilitation2005; 19: 283-289.

25. Ahmed HI, El-Negamy EH, Khaled AO and Hendawy AS. Effect of ankle weight during gait training on dorsiflexors strength in hemiparetic children. Med. J. Cairo Univ., Vol.87, No.6, September:3619-3624,2019.

26. Shamay SN, Hui-Chan CW and Shamay SN. Contribution of ankle dorsiflexor strength to walking endurance in people with spastic hemiplegia after stroke. Arch Phys Med Rehabil.2012 Jun;93(6):1046-51. 
Table 1. Comparison of subject characteristics between control and study groups

\begin{tabular}{|l|l|l|l|}
\hline & Control group $(\mathrm{n}=20)$ & Study group $(\mathrm{n}=20)$ & $\mathrm{p}$ value \\
\cline { 2 - 4 } & Mean \pm SD & Mean \pm SD & \\
\hline Age (years) & $4.2 \pm 1.06$ & $4.45 \pm 1.01$ & 0.45 \\
\hline Weight $(\mathrm{kg})$ & $17.87 \pm 2.87$ & $17.52 \pm 2.54$ & 0.68 \\
\hline Height $(\mathrm{cm})$ & $96.85 \pm 10$ & $95.1 \pm 8.22$ & 0.54 \\
\hline GMFCS, median & 2 & 2 & 0.19 \\
\hline Sex distribution & & & \multirow{2}{*}{0.51} \\
\hline Girls & $7(35 \%)$ & $9(45 \%)$ & \\
\hline Boys & $13(65 \%)$ & $11(55 \%)$ & \multirow{2}{*}{0.34} \\
\hline Spasticity grades & & & \\
\hline Grade I & $9(45 \%)$ & $12(60 \%)$ & \\
\hline Grade I+ & $11(55 \%)$ & $8(40 \%)$ & \\
\hline
\end{tabular}

$\mathrm{SD}$, standard deviation; $\mathrm{p}$ value, probability value

Table 2. Mean degree of genu recurvatum and tibialis anterior and hamstring muscles strength pre and post treatment of the control and study groups

\begin{tabular}{|c|c|c|c|c|c|}
\hline & $\begin{array}{l}\text { Control group } \\
(\mathrm{n}=20)\end{array}$ & $\begin{array}{l}\text { Study group } \\
(n=20)\end{array}$ & & & \\
\hline & Mean \pm SD & Mean \pm SD & $\mathrm{MD}$ & t- value & p value \\
\hline \multicolumn{6}{|c|}{ Genu recurvatum (degrees) } \\
\hline Pre treatment & $11.75 \pm 1.71$ & $11.7 \pm 1.83$ & 0.05 & 0.08 & 0.92 \\
\hline Post treatment & $10.6 \pm 1.6$ & $9.47 \pm 1.5$ & 1.13 & 2.28 & 0.02 \\
\hline $\mathrm{MD}$ & 1.15 & 2.23 & & & \\
\hline$\%$ of change & 9.79 & 19.06 & & & \\
\hline \multirow[t]{2}{*}{$\mathrm{t}$ - value } & 14.03 & 21.07 & & & \\
\hline & $p=0.001$ & $p=0.001$ & & & \\
\hline \multicolumn{6}{|c|}{ Tibialis anterior strength $(\mathrm{kg})$} \\
\hline Pre treatment & $2.23 \pm 0.67$ & $2.31 \pm 0.58$ & -0.08 & -0.37 & 0.71 \\
\hline Post treatment & $2.6 \pm 0.64$ & $3.06 \pm 0.57$ & -0.46 & -2.37 & 0.02 \\
\hline $\mathrm{MD}$ & -0.37 & -0.75 & & & \\
\hline$\%$ of change & 16.59 & 32.47 & & & \\
\hline \multirow[t]{2}{*}{$\mathrm{t}$ - value } & -10.9 & -29.24 & & & \\
\hline & $p=0.001$ & $p=0.001$ & & & \\
\hline \multicolumn{6}{|c|}{ Hamstring strength $(\mathrm{kg})$} \\
\hline Pre treatment & $2.7 \pm 0.68$ & $2.85 \pm 0.82$ & -0.15 & -0.64 & 0.52 \\
\hline Post treatment & $3.09 \pm 0.64$ & $3.6 \pm 0.73$ & -0.51 & -2.33 & 0.02 \\
\hline $\mathrm{MD}$ & -0.39 & -0.75 & & & \\
\hline$\%$ of change & 14.44 & 26.32 & & & \\
\hline \multirow[t]{2}{*}{ t-value } & -19.12 & -26.1 & & & \\
\hline & $p=0.001$ & $p=0.001$ & & & \\
\hline
\end{tabular}

$\mathrm{SD}$, standard deviation; MD, mean difference; $p$-value, probability value

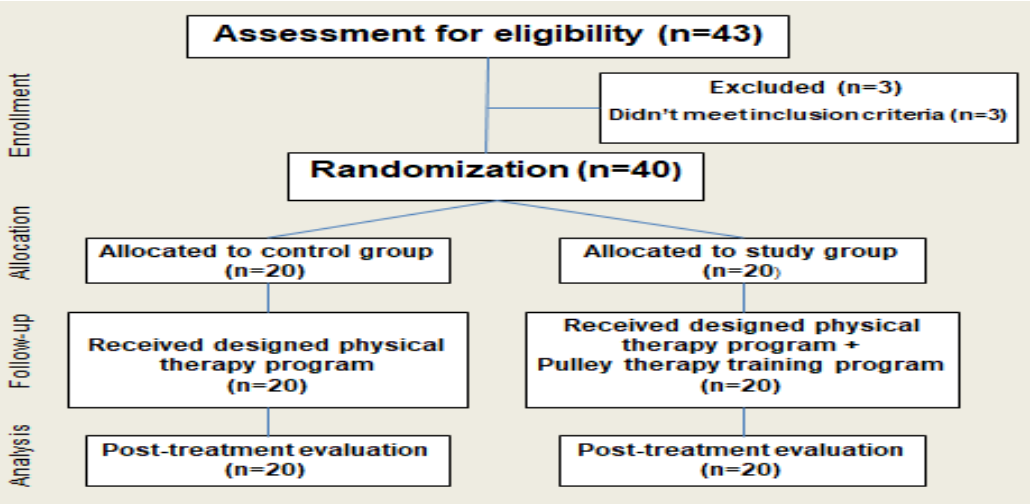

Figure 1. Participants' flow chart 